\title{
Carcinosarcoma of the stomach: A case report and review of the literature
}

\author{
Tomislav Randjelovic, Branka Filipovic, Darko Babic, Vesna Cemerikic, Branislav Filipovic
}

Tomislav Randjelovic, Department of Gastrointestinal Surgery, Clinical and Hospital Center "Bezanijska Kosa", Belgrade, Serbia Branka Filipovic, Department of Gastroenterohepatology Clinical and Hospital Center "Bezanijska Kosa", Belgrade 11080, Serbia Darko Babic, Department of Pathology, Clinical and Hospital Center "Bezanijska Kosa", Belgrade 11080, Serbia

Vesna Cemerikic, Laboratory for Histopathological and Cytological Diagnostics "Histolab", Belgrade 11080, Serbia

Branislav Filipovic, Institute of Anatomy, School of Medicine, University of Belgrade, Belgrade 11080, Serbia

Correspondence to: Branka Filipovic, MD, MSc, Department of Gastroenterohepatology, Clinical and Hospital Center "Bezanijska Kosa", Autoput bb, Belgrade 11080, Serbia.branaf@bitsyu.net Telephone: +381-11-3010700 Fax: +381-11-3010751

Received: May 20, 2007 Revised: June 18, 2007

\begin{abstract}
Carcinosarcomas are rare, malignant, biphasic tumors. We report the case of a 62-year-old man with gastric carcinosarcoma, along with its clinical, macroscopic and histopathological features. Macroscopically, a specimen of deformed stomach was obtained that measured $200 \mathrm{~mm} \times 150 \mathrm{~mm} \times 100 \mathrm{~mm}$. A $150 \mathrm{~mm} \times 100 \mathrm{~mm} \times$ $50 \mathrm{~mm}$ exophytic tumoral mass (Borrmann type I ) was found, which involved the posterior wall from the cardia to the antrum. Histopathologically, a mixed type of malignancy was revealed: an adenocarcinoma with intestinal metaplasia, with interposed fascicles of fusiform atypical cells and numerous large, rounded and oval cells. The tumor showed positive histochemistry for cytokeratin 18, epithelial membrane antigen, carcinoembryonic antigen, chromogranin $\mathrm{A}$ and vimentin. Liver metastases were diagnosed 8 mo postoperatively, and the patient died 4 mo later. A review of the available literature is also presented.
\end{abstract}

(C) 2007 WJG. All rights reserved.

Key words: Carcinosarcoma; Histochemistry; Pathology; Stomach

Randjelovic T, Filipovic B, Babic D, Cemerikic V, Filipovic B. Carcinosarcoma of the stomach: A case report and review of the literature. World J Gastroenterol 2007; 13(41): 5533-5536

http://www.wjgnet.com/1007-9327/13/5533.asp

\section{INTRODUCTION}

Carcinosarcomas are rare, malignant, biphasic tumors. In the upper gastrointestinal tract, they are most frequently observed in the esophagus, while localization in the stomach has been less frequently reported ${ }^{[1-3]}$. We present the case of a 62-year-old man with gastric carcinosarcoma, along with its clinical, macroscopic and histopathological features.

\section{CASE REPORT}

The patient was a 62-year-old man admitted for surgery with a 2-mo history of blunt epigastric pain, nausea, loss of body weight and intermittent bleeding from the upper gastrointestinal tract. His and his family medical history was unremarkable.

Upon admission, the patient was in a forced position, bent anteriorly with a facial expression of pain. General examination revealed marked pallor, and tenderness in the epigastric region, radiating to the right side of the anterior abdominal wall. In the space of Labbé, an elastic, resistant, fixed mass was palpated. Routine laboratory parameters were found to be normal, except for markers of hypochromic anemia and inflammation: hemoglobin $100 \mathrm{~g} / \mathrm{L}$, hematocrit 26\%, mean corpuscular volume (MCV) $78 \mathrm{fL}$, iron blood level $6.8 \mu \mathrm{mol} / \mathrm{L}$, iron-binding capacity $84 \mu \mathrm{mol} / \mathrm{L}$, saturation $8 \%$, plasma fibrinogen $6.9 \mathrm{~g} / \mathrm{L}$, and erythrocyte sedimentation rate $85 \mathrm{~mm}$ at the end of the first hour (Wintrobe). The concentration of CA 72.4 was $110 \mathrm{U} / \mathrm{mL}$.

Endoscopic examination revealed an exophytic, lobulated mass that infiltrated the entire posterior wall of the stomach, from the cardia to the antrum, obturating the gastric lumen throughout. An endoscopically taken biopsy revealed signs of carcinosarcoma, with strongly expressed adenomatous and fibromatous components. Barium-based contrast radiography revealed a satisfactorily passable pyloric canal, despite the initial antral obturation. Computerized abdominal tomography (Figure 1) detected an irregular inhomogeneous, prominent tumorous formation $(120 \mathrm{~mm} \times 80 \mathrm{~mm} \times 50 \mathrm{~mm})$ in the stomach, with enlarged solitary lymph nodes of up to $2 \mathrm{~cm}$ disseminated along the minor and major gastric curvatures. The patient subsequently underwent total gastrectomy with Roux-en-Y esophagojejunostomy and extirpation of the affected lymph nodes. Macroscopically, a specimen 


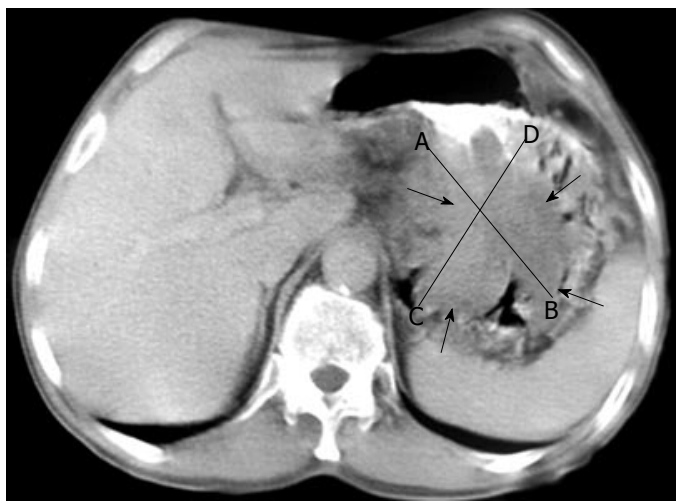

Figure 1 CT scan of the abdomen showing tumor (arrows). AB, CD: tumor diameters.

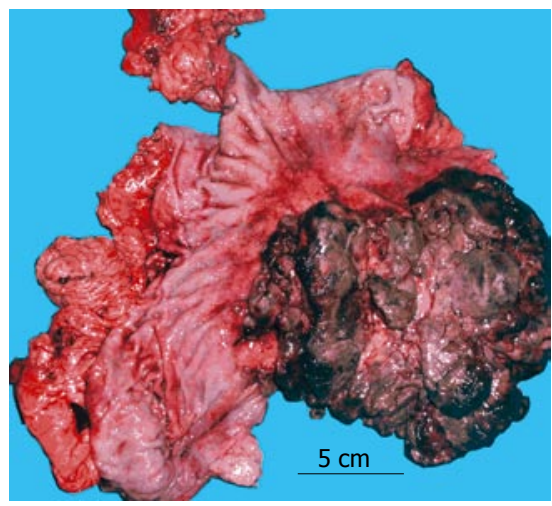

Figure 2 Macroscopic specimen obtained during surgery. Tumor underwent central necrosis, and a hemorrhagic zone is visible on the periphery.

of deformed stomach that measured $200 \mathrm{~mm} \times 150$ $\mathrm{mm} \times 100 \mathrm{~mm}$ was obtained. A $150 \mathrm{~mm} \times 100 \mathrm{~mm} \times$ $50 \mathrm{~mm}$ exophytic tumoral mass (Borrmann type I) was found, which involved the posterior wall from the cardia to the antrum. Areas of necrosis and haemorrhagia were observed in the tumor (Figure 2). The tumor did not invade the esophagus or duodenum, and metastases to other organs were not observed (TNM stage IIIA).

Histopathologically, the tumor involved all the layers of the gastric wall. The malignancy had two components, epithelial and mesenchymal. The epithelial component consisted of irregular, dilated adenomatous structures, along with a low cylindrical epithelium, with atypical, pleomorphic hyperchromatic or vesiculous nuclei and detectable nucleoli. Between the glandular formations spread the mesenchymal component, consisting of fascicles of fusiform atypical cells and numerous large, rounded and oval cells, with extremely pleomorphic, hyperchromatic nuclei. Among the aforementioned, multiple atypical, multinuclear giant cells with bizarre hyperchromatic nuclei and spotted vacuolated cytoplasm were scattered (Figure 3A and B). The epithelial component showed positive histochemistry for cytokeratin 18 (Figure 4), epithelial membrane antigen (EMA) and carcinoembryonic antigen (CEA). Chromogranin-A-positive epithelial cells were also observed. The mesenchymal component showed intensive staining for vimentin (Figure 5), although neither muscular nor neural differentiation was found. No H pylori was seen.
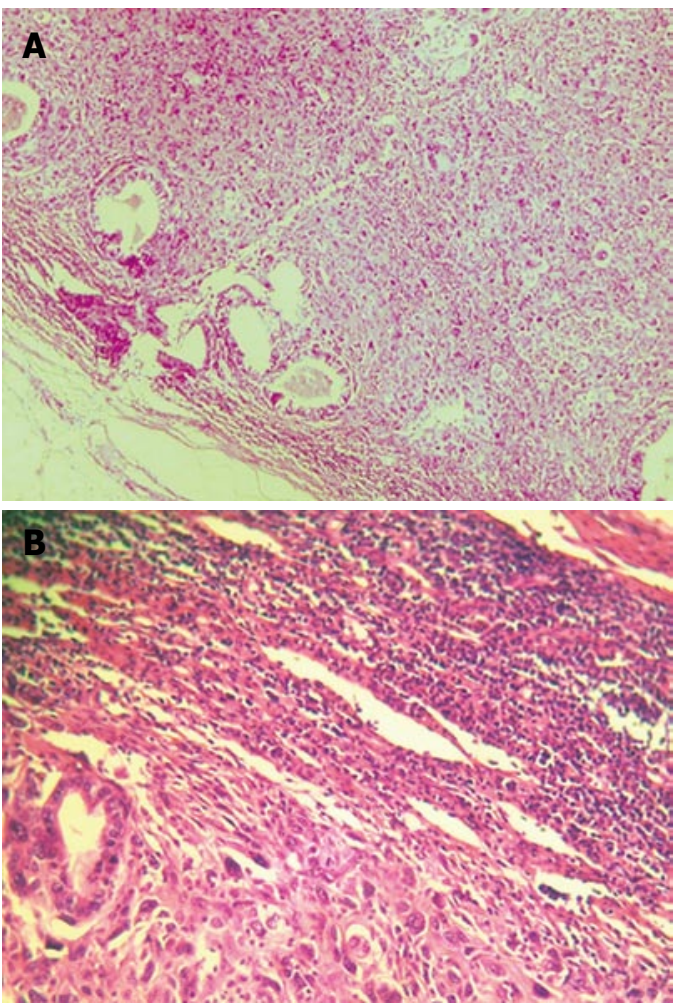

Figure 3 Massive lymph node infiltration with tumor cells. Only a few tubules can be seen on the lymph-node periphery. In between and all around, large polygonal cells are haphazardly arranged (sarcomatous component). This appearance resembles that of the main tumor mass. A: hematoxylin-eosin (HE), × 50; B: HE, $\times 100$.

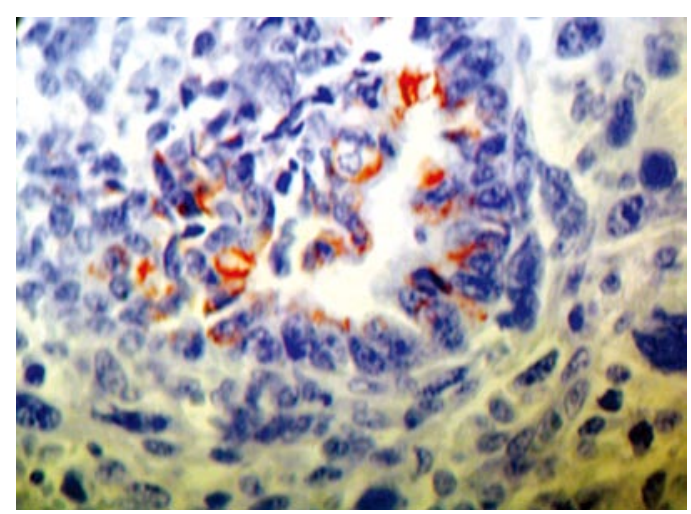

Figure 4 Cytokeratin-18-positive epithelial cells arranged in tubules $(\times 400)$.

The patient was discharged on the fifteenth postoperative day in a very well condition. Eight months after operation, liver metastases were observed on CT scanning, but his Karnofsky performance status (50) and Eastern Cooperative Oncology Group performance status (3) did not allow the administration of chemotherapy, and therefore he only received symptomatic medications. $\mathrm{He}$ died about 4 mo later.

\section{DISCUSSION}

In this paper, we presented the case of a patient with stomach carcinosarcoma, with simultaneous occurrence of moderately to well-differentiated adenocarcinoma 


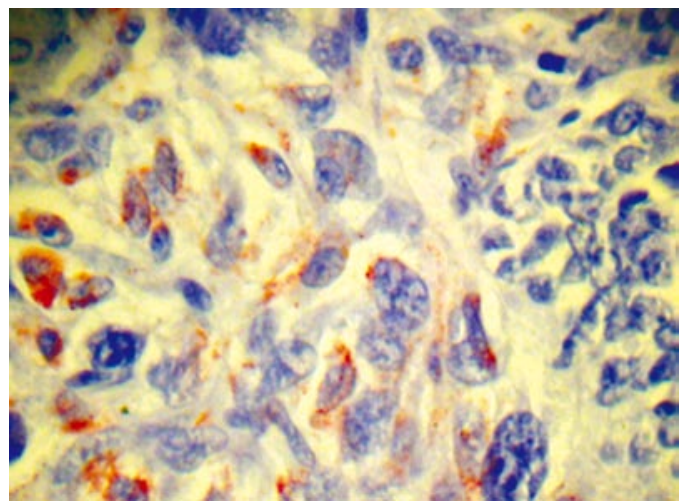

Figure 5 Vimentin-positive polygonal tumor cells $(\times 400)$.

and traces of neuroendocrine, chromogranin-A-positive elements, combined with a vimentin-positive mesenchymal component. To the best of our knowledge, this is the first case of gastric carcinosarcoma seen in this part of the world. According to the available sources, about 50 cases of gastric carcinosarcoma have been reported so far, mostly in Japan and predominantly in the male population, mostly over the age of 60 years ${ }^{[4-6]}$. An Italian group has experienced five cases of synchronous occurrence of adenocarcinoma and stromal tumor during a 10 -year period ${ }^{[7]}$. Carcinosarcomas in the stomach may be polypoid, exophytic or endophytic, with generally ulcerated surfaces, and they frequently infiltrate the gastric wall in the antral or pyloric region, and form large tumor masses ${ }^{[2,4,7]}$. Intestinal adenocarcinoma is predominant, but carcinoid and endocrinous or neuroendocrinous elements have been observed during the synchronous appearance of carcinoma and sarcoma in the stomach, while the sarcomatous component can vary between myoblastic, rhabdomyoblastic, chondroblastic and osteoblastic differ entiation $^{[2,5,6,8-11]}$. Metastasis of carcinosarcoma, however, may be entirely carcinomatous, sarcomatous or biphasic in appearance $^{[4]}$.

Immunocytochemistry seems to be the gold standard for diagnosis of carcinosarcoma, because contrast-based radiography, computerized tomography $(\mathrm{CT})$ and even endoscopy appear to be less efficient, and occasionally, even standard light microscopy is not adequate. Therefore, CEA, EMA, pancreatin, chromogranin A, CD56 and synaptophysin staining are highly specific markers for the carcinomatous components, while desmin, vimentin and $\alpha$-smooth muscle/sarcomeric actin show affinity for the sarcomatous elements ${ }^{[5,12]}$.

Therapy of carcinosarcoma is always radical and comprises partial or total gastrectomy with Rouxen-Y deviation of one of the jejunal loops, although some complications might appear in the postoperative period $^{[13,14]}$. Some experimental studies reported possible tumor reduction following treatment with methionine/ valine-depleted enteral nutrition, although its efficacy in humans is ambiguous and remains to be established ${ }^{[15]}$.

Prognosis of carcinosarcoma in the stomach is poor ${ }^{[6]}$, and patients with gastric endocrine cell carcinoma have a poorer prognosis than those with other types of gastric carcinoma. The mean survival period is estimated to be $10-15 \mathrm{mo}$, and overall tumor recurrence in the first postoperative year is greater than $50 \%{ }^{[5,9,12]}$.

With respect to the histogenesis of carcinosarcoma, two hypotheses have been proposed. Some authors have suggested that carcinosarcoma is derived from a single totipotential stem cell that has the ability to pursue both epithelial and mesenchymal differentiation ${ }^{[14]}$. There is no strong evidence that $H$ pylori infection influences the appearance of carcinosarcoma ${ }^{[7,14]}$.

In conclusion, carcinosarcoma of the stomach is a rare tumor with high malignant potential, often of unclear etiology. At present, no clinical tests are available for early diagnosis (MRI, barium-based gastrography). The gold standard for definitive diagnosis is immunohistochemical staining of endoscopic biopsy. The possibilities for therapy are confined to radical Roux-en-Y esophagojejunostomy, and recurrence of the tumor can be expected within the first postoperative year.

\section{REFERENCES}

1 Kanamoto A, Nakanishi Y, Ochiai A, Shimoda T, Yamaguchi H, Tachimori Y, Kato H, Watanabe H. A case of small polypoid esophageal carcinoma with multidirectional differentiation, including neuroendocrine, squamous, ciliated glandular, and sarcomatous components. Arch Pathol Lab Med 2000; 124: 1685-1687

2 Yamazaki K. A gastric carcinosarcoma with neuroendocrine cell differentiation and undifferentiated spindle-shaped sarcoma component possibly progressing from the conventional tubular adenocarcinoma; an immunohistochemical and ultrastructural study. Virchows Arch 2003; 442: 77-81

3 Insabato L, Di Vizio D, Ciancia G, Pettinato G, Tornillo L, Terracciano L. Malignant gastrointestinal leiomyosarcoma and gastrointestinal stromal tumor with prominent osteoclast-like giant cells. Arch Pathol Lab Med 2004; 128: 440-443

4 Kayaselcuk F, Tuncer I, Toyganözü Y, Bal N, Ozgür G. Carcinosarcoma of the stomach. Pathol Oncol Res 2002; 8: 275-277

5 Teramachi K, Kanomata N, Hasebe T, Ishii G, Sugito M, Ochiai A. Carcinosarcoma (pure endocrine cell carcinoma with sarcoma components) of the stomach. Pathol Int 2003; 53: 552-556

6 Nakayama $\mathbf{Y}$, Murayama $H$, Iwasaki $H$, Iwanaga $S$, Kikuchi M, Ikeda S, Okada M, Iizuka Y, Iwashita A. Gastric carcinosarcoma (sarcomatoid carcinoma) with rhabdomyoblastic and osteoblastic differentiation. Pathol Int 1997; 47: 557-563

7 Maiorana A, Fante R, Maria Cesinaro A, Adriana Fano R. Synchronous occurrence of epithelial and stromal tumors in the stomach: a report of 6 cases. Arch Pathol Lab Med 2000; 124: 682-686

8 Tsuneyama K, Sasaki M, Sabit A, Yokoi K, Arano Y, Imai T, Nakanuma Y. A case report of gastric carcinosarcoma with rhabdomyosarcomatous and neuroendocrinal differentiation. Pathol Res Pract 1999; 195: 93-97; discussion 98

9 Matsui K, Jin XM, Kitagawa M, Miwa A. Clinicopathologic features of neuroendocrine carcinomas of the stomach: appraisal of small cell and large cell variants. Arch Pathol Lab Med 1998; 122: 1010-1017

10 Pase F, Galassi A, Tormen D, Missaglia C, Petrelli G, D'Amore ES. Composite tumour of the stomach: a case report and review of the literature. Chir Ital 2005; 57: 99-102

11 Kuroda N, Oonishi K, Iwamura S, Ohara M, Hirouchi T, Mizumo K, Miyazaki E, Enzan H. Gastric carcinosarcoma with neuroendocrine differentiation as the carcinoma component 
and leiomyosarcomatous and myofibroblastic differentiation as the sarcomatous component. APMIS 2006; 114: 234-238

12 Sato Y, Shimozono T, Kawano S, Toyoda K, Onoe K, Asada Y, Hayashi T. Gastric carcinosarcoma, coexistence of adenosquamous carcinoma and rhabdomyosarcoma: a case report. Histopathology 2001; 39: 543-544

13 Chen YP, Yang JS, Liu DT, Chen YQ, Yang WP. Longterm effect on carcinoma of esophagus of distal subtotal gastrectomy. World J Gastroenterol 2004; 10: 626-629

14 Liu SW, Chen GH, Hsieh PP. Collision tumor of the stomach: a case report of mixed gastrointestinal stromal tumor and adenocarcinoma. J Clin Gastroenterol 2002; 35: 332-334

15 He YC, Cao J, Chen JW, Pan DY, Zhou YK. Influence of methionine/valine-depleted enteral nutrition on nucleic acid and protein metabolism in tumor-bearing rats. World J Gastroenterol 2003; 9: 771-774

S- Editor Liu Y L- Editor Kerr C E- Editor Ma WH 